\title{
LigaSure in Total Thyroidectomy
}

\author{
Calogero Cipolla, Giuseppa Graceffa, Luigi Sandonato, Salvatore Fricano, Salvatore Vieni, \\ and MARio A. LATTERI \\ Department of Oncology, Division of General and Oncological Surgery, University of Palermo, AOU Policlinico Paolo Giaccone, \\ Palermo, Italy
}

\begin{abstract}
Purpose. LigaSure is a bipolar diathermy system, which achieves vessel sealing with reduced thermal spread. The device has been used successfully in abdominal operations and because of its features, it has been applied recently in thyroid surgery to minimize the risk of complications such as laryngeal nerve palsy and hypocalcemia, and also to reduce the operating time.

Methods. Between June and December 2005, we performed total thyroidectomy for various disorders in 105 patients. We used the LigaSure diathermy system in 53 patients and traditional hemostatic procedures in the other 52. We evaluated the demographic features, histopathological diagnosis, operating times, and relevant postoperative complications.

Results. The two study groups had similar demographic and histopathological features. The mean operating time \pm SD was not significantly shorter in the LigaSure group than in the traditional group (104 \pm 12.7 vs $110 \pm$ $15.6 \mathrm{~min}$ ). None of the patients in either group suffered hemorrhage, definitive hypocalcemia, or definitive laryngeal nerve palsy. Transient hypocalcemia and transient laryngeal nerve palsy developed in $7.54 \%$ and in $1.88 \%$, respectively, of the patients in the LigaSure group, and in $7.69 \%$ and $1.92 \%$, respectively, of the patients in the traditional group; these differences were not significant.

Conclusions. LigaSure is a safe and effective method of hemostasis control, but it did not reduce the incidence of complications or operating times compared with traditional hemostatic procedures; moreover, the operative costs were higher.
\end{abstract}

Key words LigaSure $\cdot$ Thyroidectomy $\cdot$ Complications

Reprint requests to: C. Cipolla, Via Pietro Di Novo 5, 90018 Termini Imerese (PA), Italy

Received: October 5, 2006 / Accepted: March 9, 2007

\section{Introduction}

Thyroidectomy is one of the most frequently performed operations in the field of general surgery. It involves meticulous devascularization of the thyroid gland, which has one of the richest blood supplies of all organs. The gold standard for intraoperative hemostasis in thyroid surgery is suture ligation with bipolar or monopolar electrocoagulation for smaller vessels. ${ }^{\square}$ However, the use of electrocoagulation to control bleeding carries a risk of damage to the surrounding structures because of the lateral heat dispersion. 2 Recently, several alternative methods of hemostasis, such as the harmonic scalpel and laser techniques, have been tested in thyroid surgery with encouraging results $\frac{3-6}{2}$ but few reports have been published on LigaSure in this field.

Apart from its hemostatic efficiency and safety, LigaSure may minimize the risk of damage to the parathyroid glands and to the recurrent laryngeal nerve, because it disperses less heat to the surrounding tissue than classic bipolar or monopolar electrocoagulation methods. We conducted this study to compare LigaSure with traditional hemostatic procedures in total thyroidectomy, focusing on operating times and potential relevant postoperative complications. In our experience, total thyroidectomy is the treatment of choice, not only for thyroid carcinoma, but for most benign thyroid diseases, because it prevents disease relapse and the need for repeated surgery with its related higher rate of complications.

\section{Patients and Methods}

The subjects of this study were 105 patients who underwent total thyroidectomy in our surgical department between June and December 2005. Exclusion criteria included diseases involving the vocal cords, secondary thyroid surgery, previous operations on the neck, and 
the need for lymph node dissection. All patients had preoperative workup consisting of clinical examination, thyroid ultrasound and scintigraphy, cytological sampling with fine-needle aspiration, thyroid hormone assay, and optic fiber laryngoscopy to observe vocal cord motility. The same surgical team operated on all patients. Total thyroidectomy is indicated when an increase in glandular volume causes compressive symptoms, when drug therapy has proved unsatisfactory, and above all, for chronic thyroiditis with suspected neoplastic degeneration of one or more nodules.

Patients were divided into two groups according to the method of hemostasis used during total thyroidectomy: LigaSure $(n=53)$ or traditional hemostatic methods of knot-tying and bipolar electrocoagulation $(n=52)$. The basis of the study and the surgical procedures were explained to each patient, whose inclusion in one group or the other was based on their choice, age, and on the preoperative diagnostic assessment, with the aim of obtaining two relatively homogeneous groups for the most accurate results possible. Standard total thyroidectomy was performed in all patients. After a skin collar incision, subplatysmal flaps were prepared and the strap muscles were divided along the midline until the thyroid gland was exposed and dislocated. The upper, middle, and lower vascular peduncles were then isolated and sectioned, with the smaller vascular structures in the ligament of Berry. During this procedure, extreme care was taken to identify and safeguard the recurrent laryngeal nerves and the parathyroid glands; furthermore, in the LigaSure group, manual ligatures were inserted if the point of the instrument was in contact with the recurrent nerves or the parathyroid glands. At the conclusion of thyroidectomy, the operative field was irrigated and a small closed suction drainage tube was inserted. The strap muscles were then drawn together with absorbable, 2-0 interrupted sutures; the platysma and the subcutaneous tissue were sutured with absorbable, 3-0 interrupted sutures; and skin closure was done with non-absorbable 3-0 subcutaneous sutures. These latter stitches were removed on postoperative day (POD) 10.
The operating time was defined as the time from the skin incision to the final suture in skin closure. Hypoparathyroidism was diagnosed by clinical symptoms requiring replacement therapy of calcium and vitamin $\mathrm{D}$, after diagnostic confirmation by an assay of serum values of calcemia and phosphatemia. All patients were followed up 30 days after surgery, with optic fiber laryngoscopy, to assess vocal cord function. Both hypothyroidism and recurrent palsy were considered permanent if they had not resolved 6 months after surgery. Statistical analysis was done by the $\chi^{2}$ test, with Yates' correction where appropriate.

\section{Results}

The 53 patients in the LigaSure group consisted of 10 men and 43 women, ranging in age from 27 to 77 years (mean $50.1 \pm 11.4$ years), while the 52 patients in the traditional group consisted of 9 men and 43 women, ranging in age from 17 to 75 years (mean $50.5 \pm 14.3$ years).

The histopathological diagnoses were as follows: normofunctional multinodular goiter in 33 patients from the LigaSure group and 31 from the traditional group; hyperfunctional nodular goiter in 4 patients from the LigaSure group and 5 from the traditional group; Graves' disease in 3 patients from the LigaSure patients and 2 from the traditional group; autoimmune lymphocytic thyroiditis in 8 patients from the LigaSure group and 8 from the traditional group; and differentiated carcinoma of the thyroid in 5 patients from the LigaSure group and 6 from the traditional group (Table 1).

The mean operating times were $104 \pm 12.7$ min (range 80-145 min) in the LigaSure group and $110 \pm 15.6 \mathrm{~min}$ (range 85-135 min) in the traditional group, without a significant difference. The reduction in mean operating time when the LigaSure was used instead of traditional methods of hemostasis was only 6 min. The mean intraoperative blood loss was $58 \pm 38.2 \mathrm{ml}$ (range $35-115 \mathrm{ml}$ ) in the LigaSure group and $61 \pm 37.5 \mathrm{ml}$ (range $35-125 \mathrm{ml}$ ) in the traditional group, without a significant difference.

Table 1. Demographic and histopathological features of the patients undergoing total thyroidectomy with LigaSure or traditional hemostatic methods

\begin{tabular}{lcc}
\hline & LigaSure group & Traditional group \\
\hline Age (years) & $22-77$ & $17-75$ \\
M/F & mean $50.1 \pm 11.4$ & mean $50.5 \pm 14.3$ \\
Normofunctional multinodular goiter & $10 / 43$ & $9 / 43$ \\
Hyperfunctional multinodular goiter & 33 & 31 \\
Graves' disease & 4 & 5 \\
Autoimmune lymphocytic thyroiditis & 3 & 2 \\
Differentiated thyroid carcinoma & 8 & 8 \\
\hline
\end{tabular}


Table 2. Operating times, weight of the thyroid gland, intraoperative blood loss, drainage volume, and complications in the two groups

\begin{tabular}{lccc}
\hline & LigaSure group & Traditional group & $P$ \\
\hline Operating time (min) & $80-145$ & $85-135$ & NS \\
Gland weight (g) & mean $104 \pm 12.7$ & mean $110 \pm 15.6$ & \\
& $40-205$ & $45-220$ & NS \\
Intraoperative blood loss (ml) & $35-115$ & $120 \pm 36.1$ & \\
& $58 \pm 38.2$ & $35-125$ & NS \\
Drainage volume (ml) & $55-125$ & $61 \pm 37.5$ & \\
& mean $89 \pm 12.4$ & mean $86 \pm 12.6$ & NS \\
Hemorrhage & 0 & 0 & NS \\
Definitive hypoparathyroidism & 0 & 0 & NS \\
Transient hypoparathyroidism & $4(7.5 \%)$ & $4(7.7 \%)$ & NS \\
Definitive laryngeal nerve palsy & 0 & 0 & NS \\
Transient laryngeal nerve palsy & $1(1.9 \%)$ & $1(1.9 \%)$ & NS \\
\hline
\end{tabular}

In both groups, the suction drainage was removed on postoperative day (POD) 1 . The mean drainage output was $89 \pm 12.4 \mathrm{ml}$ (range $55-125 \mathrm{ml}$ ) in the LigaSure group and $86 \pm 12.6 \mathrm{ml}$ (range $50-105 \mathrm{ml}$ ) in the traditional group, without significance.

There were no significant differences between the two groups in the weight of the excised thyroid gland at histopathological examination or in the incidence of postoperative complications. None of the patients in either group required reoperation for bleeding, and none suffered permanent hypoparathyroidism or recurrent palsy. Transient recurrent palsy developed in one $(1.9 \%)$ patient from the LigaSure group and one $(1.9 \%)$ from the traditional group. Symptomatic transient hypoparathyroidism was confirmed by low serum levels of calcium in four $(7.5 \%)$ patients from the LigaSure group and four $(7.7 \%)$ from the traditional group, without significance Table 2). All patients were discharged from hospital on POD 2.

\section{Discussion}

Total thyroidectomy is the treatment of choice for many thyroid diseases. This operation is performed frequently in regions such as ours, where goiter is an endemic disease, and many affected people seek surgical treatment only after the goiter has become voluminous. As with any surgical procedure, the surgeon tries to minimize the risk of complications and to ensure that the operation proceeds as smoothly and rapidly as possible.

For some time, LigaSure has been used successfully in numerous abdominal, urological, and gynecological operations and many clinical studies have confirmed its efficiency and safety; not only for achieving hemostasis, but also for reducing the operating time, particularly in laparoscopic procedures. ${ }^{3-7}$ The LigaSure vessel sealing system, developed by Valleylab (Boulder, CO, USA), is a hemostatic method offering a consistent permanent autologous seal to veins, arteries, and tissue bundles of up to $7 \mathrm{~mm}$ in diameter. It consists of an electrosurgical generator and a handpiece with a ratcheted scissor mechanism. When the tissue is grasped and compressed by the instrument, the response generator senses the density of the tissue bundle, and the generator computer automatically adjusts the amount of energy to be delivered. This form of energy denatures collagen and elastin within the vessel wall and surrounding connective tissue. The added strong pressure applied by the instrument causes the denatured collagen and elastin to reform with the vessel walls in apposition. When sealing is complete, the microprocessor-controlled feedback automatically terminates the pulse. After the instrument is removed, the seal is visible as a translucent seal zone, which can be safely divided ${ }^{8-10}$ Sealing is achieved with minimal sticking and charring; thus, a limited amount of energy is delivered, resulting in a minimal thermal spread to adjacent tissues, of approximately 0.5 to $2 \mathrm{~mm}$

Since the effective hemostasis and limited heat spread may prevent damage to the parathyroid glands and the recurrent laryngeal nerve, the use of LigaSure has recently been extended to thyroid surgery. This is also due to the commercial availability of a much small handpiece $(16.5 \mathrm{~cm})$ than those used in abdominal surgery. However, the reported results of using LigaSure in thyroid surgery have been fairly discordant. Several authors reported a reduction in complications such as transient recurrent palsy and transient hypoparathyroidism and shorter operating times, $\frac{12}{1}$ whereas others reported a shorter operating time but no change in the incidence of complications $\frac{13-15}{5}$ Some authors even reported no difference in operating times or the incidence of complications between LigaSure and traditional hemostatic methods $\frac{16}{6}$ 
We began using LigaSure in thyroidectomy in 2002. In our previous series of 67 total thyroidectomies performed with LigaSure, we reported only a slightly lower incidence of complications than that in 579 total thyroidectomies performed with traditional hemostatic techniques ${ }^{17}$ Because our first experience with LigaSure convinced us that the method was simple, easy to learn, and technically straightforward, we decided to verify our initial results by conducting a comparative prospective study of patients scheduled to undergo total thyroidectomy. After obtaining informed consent, 105 patients were enrolled in the study and divided into two groups according to whether the LigaSure method or traditional hemostatic suturing and bipolar electrocoagulation was used. We then compared the incidence of complications and operating times between the two methods. Our analysis confirmed that LigaSure was an effective, safe, and easy-to-use hemostatic method, but there were no significant differences in the incidence of severe complications or operating times between the two methods. Moreover, we found that although the ligature of the upper and of the lower thyroid vessels seemed to be easier than and at least as fast as conventional knot-tying, meticulous ligation of the numerous small vessels with LigaSure was not as easy because of the size of the instrument tip, which was too large to use on such small vessels. In accordance with several other reports 16 we do not think that the LigaSure method is suitable for the precise surgical maneuvers required for operations in the vicinity of extremely delicate structures such as the recurrent nerves. Furthermore, the method is not very precise or reliable for the coagulation of tiny thyroid vessels. In fact, we had to ligate the tiny vessels by hand in our patients who underwent total thyroidectomy with the LigaSure method when the tip of the handpiece was in contact with the recurrent nerves or the parathyroid glands.

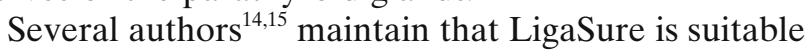
for use in hemithyroidectomy, since it makes separating the thyroid gland tissue easier than traditional methods of ligation, thereby reducing the operating time to about $20 \mathrm{~min}$. We do agree that the device may be faster and more efficient in the early phases of the operation: for coagulating the external jugular veins, for preparing flaps by separating the platysma, and for separating the strap muscles along the linea alba.

A final consideration concerns the high cost of the device: about $18000 €$ for the generator and $600 €$ for the scissor mechanism. In this regard, the cost/benefit ratio of a thyroidectomy performed with the LigaSure method is not as good as that of a thyroidectomy performed with traditional hemostatic techniques.
In conclusion, although LigaSure is without doubt an extremely efficient and safe hemostatic method for use in thyroidectomy, it does not ensure a lower incidence of complications or shorter operating times and it is very expensive. Thus, we conclude that the LigaSure method does not offer any real advantage over traditional methods of hemostasis in total thyroidectomy.

\section{References}

1. Gluckman JL. Total thyroidectomy. In: Bailey BJ, Calhoun KH, editors. Atlas of head and neck surgery: otolaryngology, 2nd ed. Philadelphia: Lippincott Williams \& Wilkins; 2001. p. 228-9.

2. Hambley R, Hebda PA, Abell E, Cohen BA, Jegasothy BV. Wound healing of skin incisions produced by ultrasonically vibrating knife, scalpel, electrosurgery, and carbon dioxide laser. J Dermatol Surg Oncol 1988;14:1213-7.

3. Siperstein AE, Berber E, Morkoyun E. The use of the harmonic scalpel vs conventional knot tying for vessel ligation in thyroid surgery. Arch Surg 2002;137:137-42.

4. Voutilainen PE, Haapiainen RK, Haglund CH. Ultrasonically activated shears in thyroid surgery. Am J Surg 1998;175:491-3.

5. Voutilainen PE, Haglund $\mathrm{CH}$. Ultrasonically activated shears in thyroidectomies: a randomized trial. Ann Surg 2000;231:322-8.

6. Meurisse M, Defechereux T, Maweja S, Degauque C, Vandelaer M, Hamoir E. Evaluation of the Ultracision ultrasonic dissector in thyroid surgery: prospective randomized study. Ann Chir 2000;125:468-72.

7. Dubuc-Lissoir J. Use of a new energy-based vessel ligation device during laparoscopic gynecologic oncologic surgery. Surg Endosc 2003;17(3):466-8.

8. Heniford BT, Matthews BD, Sing RF, Backus C, Pratt B, Greene FL. Initial results with an electrothermal bipolar vessel sealer. Surg Endosc 2001;15(8):799-801.

9. Kennedy JS, Stranahan PL, Taylor KD, Chandler JG. High-burststrength, feedback-controlled bipolar vessel sealing. Surg Endosc 1998;12:876-8.

10. Harold KL, Pollinger H, Matthews BD, Kercher KW, Sing RF, Heniford BT. Comparison of ultrasonic energy, bipolar thermal energy, and vascular clips for the hemostasis of small-, medium-, and large-sized arteries. Surg Endosc 2003;17(8):1228-30.

11. Stranahan PL, Buysse SP, Ryan TP, Pearce JA, Thomsen S, Sieve $\mathrm{VD}$, et al. Healing process and histologic evaluation following use of bipolar energy for vessel sealing. Boulder, CO: Valleylab, a Division of Tyco Healthcare Group LP; 1999.

12. Petrakis IE, Kogerakis NE, Lasithiotakis KG, Vrachassotakis N, Chalkiadakis GE. Ligasure versus clamp-and-tie thyroidectomy for benign nodular disease. Head Neck 2004;26:903-9.

13. Lachanas VA, Prokopakis EP, Mpenakis AA, Karatzanis AD, Velegrakis GA. The use of Ligasure Vessel Sealing System in thyroid surgery. Otolaryngol Head Neck Surg 2005;132:487-9.

14. Kirdak T, Korun N, Ozguc H. Use of ligasure in thyroidectomy procedures: results of a prospective comparative study. World J Surg 2005;29:771-4.

15. Shen WT, Baumbusch MA, Kebebew E, Duh QY. Use of the electrothermal vessel sealing system versus standard vessel ligation in thyroidectomy. Asian J Surg 2005;28(2):86-9.

16. Kiriakopoulos A, Dimitrios T, Dimitrios L. Use of diathermy system in thyroid surgery. Arch Surg 2004;139:997-1000.

17. Sandonato L, Cipolla C, Graceffa G, Fricano S, Li Petri S, Prinzi $\mathrm{G}$, et al. Bipolar electrothermic coagulation (Ligasure bipolar vessel sealing system) in thyroid surgery. Chir Ital 2003;55:411-5. 